\title{
Circadian Modulation on T-wave Alternans Activity in Chronic Heart Failure Patients
}

\author{
Alba Martín-Yebra ${ }^{1,2}$, Enrico G Caiani ${ }^{1}$, Pablo Laguna ${ }^{2,3}$, Violeta Monasterio ${ }^{4}$, \\ Juan Pablo Martínez,3 \\ ${ }^{1}$ Dipartimento di Elettronica, Informazione e Bioingegneria, Politecnico di Milano, Milan, Italy \\ ${ }^{2}$ BSICoS Group, Aragón Institute of Engineering Research, IIS Aragón, Universidad de Zaragoza, \\ Zaragoza, Spain \\ ${ }^{3}$ CIBER en Bioingeniería, Biomateriales y Nanomedicina (CIBER-BBN), Zaragoza, Spain \\ ${ }^{4}$ Universidad San Jorge, Villanueva de Gállego, Spain
}

\begin{abstract}
Average TWA activity has been shown to be an independent predictor of sudden cardiac death (SCD) in chronic heart failure (CHF) patients. However, the influence of circadian rhythms on TWA remains understudied. In this work, we assessed circadian TWA changes in a CHF population and evaluated whether the prognostic value of TWA indices is sensitive to the circadian pattern. Holter ECG recordings from 626 consecutive CHF patients (52 SCD) were analyzed. The index of average alternans (IAA), quantifying the average TWA level, was measured in 4 consecutive 6-hour intervals using a multilead fully-automated method. Survival analysis was performed considering SCD as an independent endpoint. IAA changed along the day, with statistically significant lower values during the night than during daytime. This pattern is similar to the one observed in the mean heart rate (HR). However, a low correlation ( $r=.18)$ was found between IAA and HR in windows of 128 beats. After dichotomization of patients based on the third quartile of IAA indices, IAA indices computed between hours 06-12 $\left(I A A_{06-12}\right)$ and 18-24 (IAA $\left.{ }_{18-24}\right)$ successfully predicted SCD (Hazard Ratio, HaR:2.34(1.33-4.13) per $\mu$ V, and HaR:1.87(1.04-3.36) per $\mu V$, respectively). In conclusion, circadian variation should be considered for SCD risk prediction.
\end{abstract}

\section{Introduction}

A great deal of deaths in patients with mild-to-moderate chronic heart failure (CHF) are represented by sudden cardiac deaths (SCD), most of them being as a consequence of ventricular tachyarrhythmias. In this context, implantable cardioverter-defibrillators (ICD) have been shown to be highly effective at terminating these life threatening arrhythmias. However, the cost-effectiveness of this therapy is still low and identifying patients who would benefit the most from ICD therapy remains a clinical challenge.

T-wave alternans (TWA), a consistent beat-to-beat alternation in the amplitude or morphology of the ST segment and/or the $\mathrm{T}$ wave, reflects temporal and spatial heterogeneity of ventricular repolarization. It is presently regarded as a noninvasive risk marker for identifying patients at risk for SCD and ventricular vulnerability [1]. Although the long-term averaging of TWA activity in ambulatory recordings has been shown to be an independent predictor of SCD in CHF [2], the influence of circadian rhythms on TWA still remains understudied.

Our aim in this study was to assess circadian TWA changes in a CHF population as well as to evaluate whether the prognostic value of TWA is sensitive to this circadian pattern.

\section{Study population}

Consecutive patients with symptomatic CHF corresponding to New York Heart Association (NYHA) classes II and III were enrolled in the multicenter MUSIC (MUerte Súbita en Insuficiencia Cardiaca) study, a prospective study designed to assess risk predictors for cardiovascular mortality in ambulatory CHF patients [3]. The study protocol was approved by institutional investigator committees and all patients gave written informed consent. The 24-hour Holter ECG recordings of 626 patients (52 victims of SCD, 63 of other cardiac causes, 25 non-cardiac deaths and 486 survivors) with sinus rhythm and aged 1889 years $(62.7 \pm 11.9$ years) were available for the present study. ECG signals were acquired by using SpiderView records (ELA Medical, Sorin Group, Paris, France) and two or three orthogonal leads (X, Y, Z) sampled at $200 \mathrm{~Hz}$ were available for each subject. Collection of clinical data for this population was reported in previous studies $[3,4]$. 
Patients were followed up every 6 months during 48 months. SCD, defined as (1) a witnessed death occurring within 60 minutes from the onset of new symptoms unless a cause other than cardiac failure was obvious, (2) an unwitnessed death ( $<24$ hours) in the absence of preexisting progressive circulatory failure or other causes of death, or (3) death during attempted resuscitation, was considered as an independent endpoint in this study. Endpoints were reviewed an classified by the MUSIC Study Endpoint Committee.

\section{Methods}

\subsection{Preprocessing}

Preprocessing of ECG recordings included heart beat detection and labelling using the Aristotle ECG analysis software [5] and linear filtering of baseline wander. Finally, the ECG was low-pass filtered (with cut-off frequency of $15 \mathrm{~Hz}$ ) to remove noise out of TWA frequency range and down-sampled.

\subsection{TWA measurement}

Index of average alternans (IAA), quantifying the average TWA level, was measured in consecutive 6-hour intervals (00.00-06.00 h; 06.00-12.00 h; 12.00-18.00 h; 18.00$24.00 \mathrm{~h}$ and denoted, respectively, as $\mathrm{IAA}_{00-06}, \mathrm{IAA}_{06-12}$, $\mathrm{IAA}_{12-18}$ and $\mathrm{IAA}_{18-24}$ ), using a multilead fully automated method based on periodic component analysis ( $\pi \mathrm{CA}) \mathrm{com}-$ bined with the Laplacian likelihood ratio method (LLRM) [2].

For each day interval, ECG signals were processed in segments of 128 consecutive beats (50\% overlapped) with additional HR stability criterion imposed, as defined in [2], in order to consider only suitable segments for automatic analysis.

For estimating the TWA waveform associated to the $\mathrm{k}^{\text {th }}$ segment, we replicated the analysis performed in [2]: the three orthogonal leads were linearly combined in order to maximize the TWA content over noise by using $\pi$ CA [6] and then, the LLRM [7] was applied in the new combined lead to estimate the TWA waveform of each segment, expressed as the median difference between ST-T complexes of even and odd beats. In vector notation, it is denoted as

$$
\mathbf{y}_{\mathrm{k}}(n)=\left[\begin{array}{lll}
y_{\mathrm{k}}(1) & \ldots & y_{\mathrm{k}}(N)
\end{array}\right]^{T}
$$

with $\mathrm{N}$ the total number of samples within the ST-T complex.

The non-visible microvolt range of TWA, sometimes comparable to the noise level, makes the TWA detection a challenging task. In those cases, the alternans waveform $\mathbf{y}_{\mathrm{k}}(n)$ may have an important noise component that should be attenuated. At this point, a novel methodological step for the computation of the TWA amplitude was included in the analysis, consisting on the phase alignment of all TWA estimated waveforms before averaging. This step is needed since the TWA component present in each $\mathbf{y}_{\mathrm{k}}(n)$ may not have the same alternating phase, and therefore might cancel out when averaging.

First, a detrended version of each $\mathbf{y}_{\mathrm{k}}$, denoted as $\mathbf{y}_{\mathrm{k}}^{\prime}(n)$ was computed:

$$
\mathbf{y}_{\mathrm{k}}^{\prime}(n)=\mathbf{y}_{\mathrm{k}}(n)-\left(a_{\mathrm{k}}+b_{\mathrm{k}} n\right)
$$

where the coefficients $a_{\mathrm{k}}$ and $b_{\mathrm{k}}$ were chosen so that $\mathbf{y}_{\mathrm{k}}(1)=0$ and $\mathbf{y}_{\mathrm{k}}(N)=0$, with the aim of eliminating any possible residual baseline component at the alternans frequency.

Then, the correlation matrix of all suitable segments, $\mathbf{R}_{\mathbf{Y}^{\prime}}$, was estimated as

$$
\mathbf{R}_{\mathbf{y}^{\prime}}=\frac{1}{\mathrm{~K}} \mathbf{Y}^{\prime} \mathbf{Y}^{\prime} T
$$

being $\mathrm{K}$ the total number of suitable segments for the analysis and $\mathbf{Y}^{\prime}$ the data matrix concatenating all suitable segments, $\mathbf{Y}^{\prime}=\left[\mathbf{y}_{1}^{\prime}(n) \ldots \mathbf{y}_{\mathrm{K}}^{\prime}(n)\right]$.

We obtained the dominant alternans waveform as the first principal component of the spatial correlation matrix, by solving:

$$
\mathbf{R}_{\mathbf{Y}^{\prime} \mathbf{W}_{1}}=\mathbf{w}_{1} \lambda_{1}
$$

where $\mathbf{w}_{1}$ corresponds to the first eigenvector and $\lambda_{1}$ the first eigenvalue of $\mathbf{R}_{\mathbf{y}^{\prime}}$.

At this point, the phase-aligned waveform, $\mathbf{y}_{\mathrm{k}}^{a}$, was estimated as:

$$
\mathbf{y}_{\mathrm{k}}^{a}=\operatorname{sign}\left(\mathbf{y}_{\mathrm{k}}^{T} \mathbf{w}_{\mathrm{l}}\right) \mathbf{y}_{\mathrm{k}}
$$

Finally, the IAA was defined as the mean value of the average waveform of all $\mathbf{y}_{\mathrm{k}}^{a}$ :

$$
\mathrm{IAA}=\frac{1}{\mathrm{~N}} \sum_{\mathrm{n}=1}^{\mathrm{N}}\left|\frac{1}{\mathrm{~K}} \sum_{\mathrm{k}=1}^{\mathrm{K}} \mathbf{y}_{\mathrm{k}}^{a}(n)\right|
$$

\subsection{Statistical analysis}

Data is presented as median $\left(25^{\text {th }} ; 75^{\text {th }}\right.$ percentiles $)$ for continuous variables, unless otherwise specified. Nonparametric Friedman test and Wilcoxon signed rank paired test with Bonferroni correction were applied to evaluate differences among time intervals. Survival analysis was performed by using Kaplan-Meier estimator and comparison of cumulative events by log-rank test. Prognostic value of TWA indices in predicting SCD was determined with univariate Cox proportional hazards analysis. For all tests, the null hypothesis was rejected for $\mathrm{p} \leq 0.05$. 

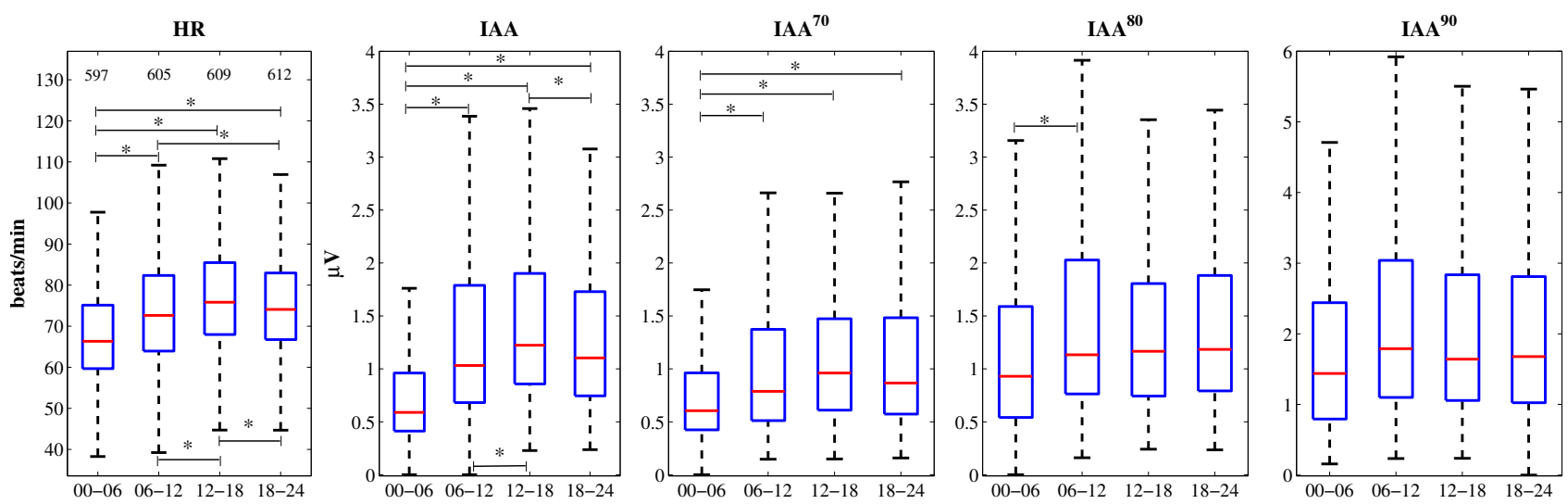

Figure 1. Distribution of HR and IAA indices for each day interval. Number of records in which IAA could be computed at each day period is indicated on left panel above the boxes.*: $\mathrm{p}<0.001$.

\section{Results}

Distribution of HR and IAA for the four defined intervals are presented in Fig. 1. Significant differences were found between the night and all daytime periods. IAA was minimal during the night period, reaching its maximum value for the interval defined from 12.00 to 18.00 $\mathrm{h}\left(\mathrm{IAA}_{00-06}=0.58(0.41 ; 0.96) \mu \mathrm{V}, \mathrm{IAA}_{12-18}=1.22(0.86 ; 1.90)\right.$ $\mu \mathrm{V}, \mathrm{p}<0.001)$. It should be noticed here that not all ECGs presented data for the total 24 hours, and consequently IAA could not be computed for each interval in all subjects (number of available recordings for each day period are indicated in Fig. 1, left panel).

The same trend was observed when considering average HR (Fig. 1, left panel). However, only a weak correlation was found between IAA and average HR $(r=0.18$, $\mathrm{p}<0.001)$ and when considering IAA HR-restricted indices (IAA ${ }^{\mathrm{X}}, \mathrm{X}=\{70,80,90\}, \mathrm{HR}$ ranging from $\mathrm{X}-10$ to $X$ beats/min) a similar pattern of variation remains visible. Additionally, an increasing tendency from IAA ${ }^{70}$ to IAA $^{90}$ in all day periods, was evidenced.

Patients were classified as TWA(+) and TWA(-) based on the third quartile of IAA indices. By setting cut points IAA $_{06-12}=1.789 \mu \mathrm{V}$ and $\mathrm{IAA}_{18-24}=1.729 \mu \mathrm{V}$, both indices successfully predicted SCD (see Table 1). Survival probability curves for $\mathrm{IAA}_{06-12}$ and $\mathrm{IAA}_{18-24}$ are shown in Fig. 2.

Table 1. Association of TWA indices with SCD death.

\begin{tabular}{l|cc} 
& $\begin{array}{c}\text { Hazard ratio } \\
(\mathbf{9 5 \%} \text { CI })\end{array}$ & p-value \\
\hline $\mathrm{IAA}_{00-06} \geq 0.961$ & $1.18(0.60,2.30)$ & 0.628 \\
$\mathrm{IAA}_{06-12} \geq 1.789$ & $\mathbf{2 . 3 4}(\mathbf{1 . 3 3 , 4 . 1 3})$ & $\mathbf{0 . 0 0 3}$ \\
$\mathrm{IAA}_{12-18} \geq 1.900$ & $1.64(0.91,2.9)$ & 0.097 \\
$\mathrm{IAA}_{18-24} \geq 1.729$ & $\mathbf{1 . 8 7}(\mathbf{1 . 0 4 , 3 . 3 6})$ & $\mathbf{0 . 0 3 5}$ \\
\hline
\end{tabular}

\section{Discussion and conclusion}

Average TWA activity computed during both the morning (from 06.00 to $12.00 \mathrm{~h}$ ) and the evening periods (18.00 to $24.00 \mathrm{~h}$ ) is associated with the risk of SCD in CHF patients. Interestingly, it coincides with the two circadian periods of elevated risk for $\mathrm{SCD}$, whose distribution has been reported to present a primary peak of incidence from 7 to 11 a.m. and a secondary peak from 5 to 7 p.m. [8]. It should be remarked that the use of indices such as IAA $\mathrm{A}_{06-12}$ and $\mathrm{IAA}_{18-24}$ indices, would considerably reduce the required time for ECG monitoring from 24 to 6 hours, which implies some advantages in the clinical practice.

The HR-restriction of IAA indices presented the same pattern for $\mathrm{IAA}^{70}$, being similar when considering $\mathrm{IAA}^{80}$ and $\mathrm{IAA}^{90}$. The increasing IAA indices from IAA ${ }^{70}$ to $\mathrm{IAA}^{90}$ are also consistent with the fact that TWA amplitudes for the same subject rises with instantaneous HR. However, only a weak correlation was found between average HR and IAA indices along the day, suggesting the influence of other HR-independent factors in IAA, which are known to be also determinant for TWA and related to higher cardiac electrical instability. Changes in autonomic neurotransmitters, with and increased activity of sympathetic nervous system during the day, especially more prominent at waking time, could be other determinant factors that significantly influence TWA [9]. Also, fluctuations in symptomatic and silent myocardial ischemia episodes, well-known to be linked to TWA phenomenon, have been reported to present this similar behavior [10]. This supports the hypothesis that IAA modulation along the day could be actually due to a circadian effect.

Automatic long-term averaging has been already shown to provide a reliable characterization of TWA in 24h-ECG recordings [2], avoiding the posterior visual verification usually needed when other methods, such as the modified 

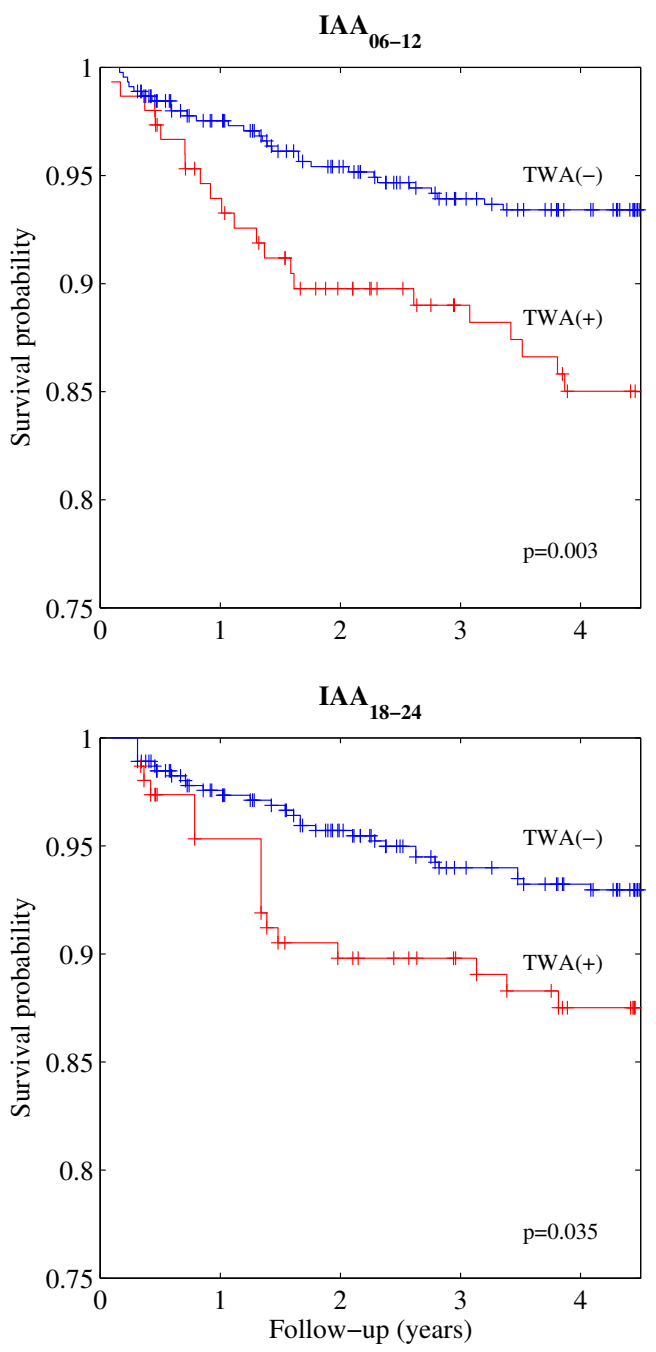

Figure 2. Survival curves for sudden cardiac death.

moving average, are used [11]. The novel approach for computing IAA indices proposed in this work, including the phase alignment of TWA waveforms before averaging, presents a more insensitive to noise solution, even in such an unfavorable condition when the TWA amplitude is at the noise level (i,e, a few microvolts).

In summary, results from a fully-automatic robust methodology for TWA analysis suggest that circadian pattern modulates the IAA index, and time of the day should be considered for SCD risk prediction.

\section{Acknowledgements}

This work was supported by CIBER in Bioengineering, Biomaterials \& Nanomedicne (CIBER-BBN) through Instituto de Salud Carlos III and FEDER (Spain), project TEC2013-42140-R funded by MINECO and FEDER, and by Gobierno de Aragón and European Social Fund (EU) through BSICoS Group (T96). The computation was performed by the ICTS NANBIOSIS, more specifically by the High Performance Computing Unit of the CIBER-BBN at the University of Zaragoza.

\section{References}

[1] Verrier RL et al. Microvolt T-wave alternans physiological basis, methods of measurement, and clinical utility - consensus guideline by International Society for Holter and Noninvasive Electrocardiology. J Am Coll Cardiol 2011;58(13):1309-24

[2] Monasterio V, Laguna P, Cygankiewicz I, et al. Average Twave alternans activity in ambulatory ECG records predicts sudden cardiac death in patients with chronic heart failure. Heart Rhythm 2012;9:383-389.

[3] Vázquez R, Bayés-Genís A, Cygankiewicz I, et al. The MUSIC risk score: a simple method for predictiong mortality in ambulatory patients with chronic heart failure. Eur Heart J 2009;30:1088 - 1096.

[4] Cygankiewicz I, Zareba W, Vázquez R, et al. Risk stratification of mortality in patients with heart failure and left ventricular ejection fraction $>35 \%$. Am J Cardiol 2009;103:1003 - 1010.

[5] Moody GB, Mark RG. Development and evaluation of a 2-lead ECG analysis program. Computers in Cardiology 1982;9:39-44.

[6] Monasterio V, Clifford GD, Laguna P, Martínez JP. A multilead scheme based on periodic component analysis for T-wave alternans analysis in the ECG. Ann Biomed Eng 2010;38(8):2532-2541

[7] Martínez JP, Olmos S. Methodological principles of $T$ wave alternans analysis: a unified framework. IEEE Trans Biomed Eng 2005;52(4):599-613

[8] Muller JE, Ludmer PL, Willich SN, et al. Circadian variation in the frequency of sudden cardiac death. Circulation 1987;75(1):131-138

[9] Verrier RL, Kumar K, Nearing BD. Basis for sudden cardiac death prediction by T-wave alternans from an integrative physiology perspective. Heart Rhythm 2009;6:416-422

[10] Nademanee K, Intarachot V, Josephson MA, et al. Circadian variation in occurrence of transient overt and silent myocardial ischemia in chronic stable angina and comparison with Prinzmental's angina in men. Am J Cardiol 1987;90:494-498

[11] Verrier RL, Nearing BD, Kwaku KF. Noninvasive sudden death risk stratification by ambulatory ECG-based T-wave alternans analysis: evidence and methodological guidelines. Ann Noninvasive Electrocardiol 2005;10:110-120

Address for correspondence:

Alba Martín

DEIB, Politecnico di Milano Piazza Leonardo da Vinci, 32, 20.133, Milano, Italy

albapilar.martin@polimi.it 